\title{
Myofibroblasts of the muscle layer stimulate the malignant potential of colorectal cancer
}

\author{
MASAFUMI TAKATSUNA ${ }^{1,3}$, SATOKO MOROHASHI ${ }^{1}$, TADASHI YOSHIZAWA ${ }^{1}$, HIDEAKI HIRAI ${ }^{1}$, \\ TOSHIHIRO HAGA ${ }^{1}$, RIE OTA ${ }^{1}$, YUNYAN WU ${ }^{1}$, HAJIME MOROHASHI ${ }^{2}$, \\ KENICHI HAKAMADA ${ }^{2}$, SHUJI TERAI ${ }^{3}$ and HIROSHI KIJIMA ${ }^{1}$
}

Departments of ${ }^{1}$ Pathology and Bioscience and ${ }^{2}$ Surgery, Hirosaki University Graduate School of Medicine, Hirosaki 036-8562; ${ }^{3}$ Division of Gastroenterology and Hepatology, Graduate School of Medical and Dental Sciences, Niigata University, Chuo-ku, Niigata 951-8510, Japan

Received January 10, 2016; Accepted February 17, 2016

DOI: 10.3892/or.2016.4932

\begin{abstract}
Myofibroblasts of colorectal cancer (CRC) have been associated with histopathological factors such as lymph node metastasis, liver metastasis and local recurrence. However, few studies have assessed the association between these malignant potentials and the myofibroblast distribution in CRC. We aimed to evaluate the relationship between clinical factors and myofibroblast distribution around CRC invasive lesions. The study included 121 cases of pT3 CRC that were diagnosed at stage II or III. Myofibroblast density of the following three histological layers was measured: the submucosa (SM), muscularis propria (MP) and subserosa (SS). We analyzed the relationship between the clinicopathological factors and myofibroblast density by studying the histopathological features of the three layers. The myofibroblast density of the MP layer was significantly higher in the groups with high-frequency lymphatic and venous invasion than the groups with low-frequency lymphatic $(\mathrm{P}<0.001)$ and venous $(\mathrm{P}<0.01)$ invasion, respectively. In the positive lymph node metastasis group, the myofibroblast density at the MP layer was significantly higher than that in the negative lymph node metastasis group $(\mathrm{P}<0.001)$. The high myofibroblast density group at the MP layer was significantly associated with poor overall survival $(\mathrm{P}<0.003)$. Our study indicated that myofibroblasts are a type of cancer-associated fibroblasts and that the myofibroblast distribution contributes to the malignant potential of CRC. Furthermore, we demonstrated that myofibroblasts
\end{abstract}

Correspondence to: Dr Satoko Morohashi, Department of Pathology and Bioscience, Hirosaki University Graduate School of Medicine, Zaifu-cho 5, Hirosaki 036-8562, Japan

E-mail: msatoko@hirosaki-u.ac.jp

Abbreviations: CRC, colorectal cancer

Key words: colorectal cancer, myofibroblast, muscularis propria, prognosis present at the MP layer play an important role in the malignant potential and poor prognosis of patients with CRC.

\section{Introduction}

Colorectal cancer (CRC) is the most common malignancy of the colon and rectum and the third most common cause of cancer-related death among men and women worldwide (1). Outcome prediction based on tumor stage reflected by the tumor node metastasis (TNM) system of the Union for International Cancer Control (UICC) is currently regarded as the standard prognostic parameter $(2,3)$. Venous and lymphatic vessel invasion are also important malignant factors of CRC (3-5). Both lymphangiogenesis and angiogenesis also play important roles as poor prognostic factors in tumorigenesis (6-8). In addition, the extracellular matrix (ECM) influences cancer proliferation, activities of invasion and metastasis by stimulating angiogenesis and lymphangiogenesis $(9,10)$.

In contrast, the relationship between CRC and myofibroblasts in the tumor microenvironment has recently attracted considerable attention. Myofibroblasts are not only known as a principal cellular component in the granulation tissue of healing wounds but are also one of the cancer stromal cells that constitute the ECM $(11,12)$. The myofibroblasts in the stroma of CRC serve an important function in promoting the desmoplastic reaction and influencing tumor invasion, microvessel density around the invasive lesion and metastatic carcinomas (13-15). Moreover, myofibroblast activation in tumor metastatic lymph nodes influences the microenvironment supporting CRC metastasis (16).

With regard to both the tumor growth and spreading of $\mathrm{CRC}$, three histological layers of the colorectum, the submucosa (SM), muscularis propria (MP) and subserosa (SS), may play important functions in the mechanical and physiological protection against invasive growth. MP is exclusively composed of smooth muscle bundles and comprises tight connective tissue, whereas SM and SS are mainly composed of loose connective tissue $(17,18)$. However, it is unclear how myofibroblasts are distributed around the CRC invasive border of these three layers as well as how the distribution is related to the malignant potential of CRC. 


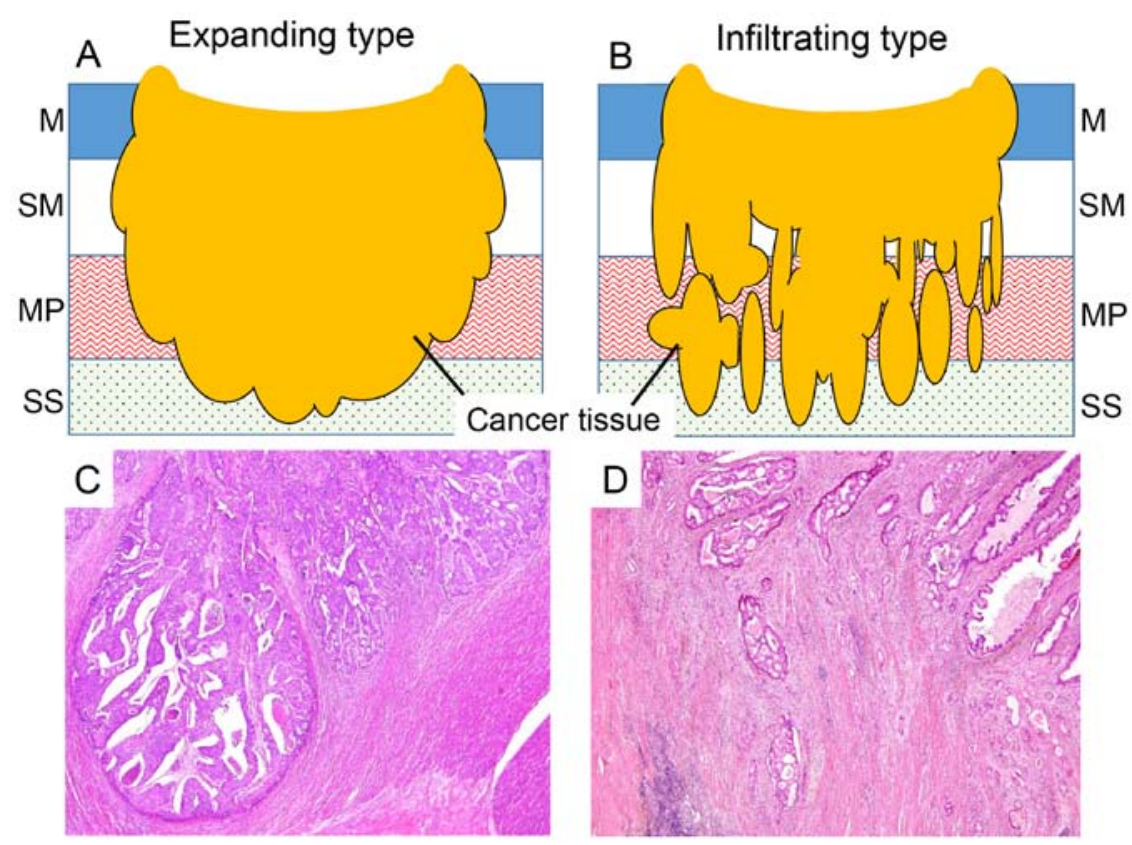

Figure 1. The expanding and infiltrating types of colorectal cancer, which invaded through the mucosa to the subserosa. The expanding type was recognised as the overall pushing growth type of adenocarcinoma and the invasive margin was clear (A). The infiltrating type was recognised as the widespread streaming form of adenocarcinoma (B). Histology of the expanding type (C) and the infiltrating type (D) around the tumor invasive lesion in the muscularis propria layer. M, mucosa; SM, submucosa; MP, muscularis propria; SS, subserosa.

In the present study, we measured the myofibroblast density of each colorectal layer using imaging analysis and investigated the association between myofibroblast distribution and clinicopathological factors such as lymph node metastasis and venous invasion. Furthermore, we showed the relationship between the myofibroblast distribution and overall survival of patients with CRC.

\section{Materials and methods}

Patients. One hundred and twenty-one patients with advanced CRC, defined as adenocarcinoma, which had invaded the SS layer of the colorectal wall (pT3), underwent surgical resection from January 2008 to December 2009 at Hirosaki University Hospital. The clinical stages of these patients were stage II or III according to the TNM classification of the UICC (2). Survival data were obtained from hospital medical charts. Cancer-specific survival was measured from the date of surgery until the date of death from CRC. None of the patients were treated with neoadjuvant chemotherapy, and none of them had synchronous multiple CRCs or synchronous metastasis to other organs.

Pathological analysis. We used surgically resected specimens that were fixed with $10 \%$ formalin, embedded in paraffin and stained with hematoxylin and eosin (H\&E) for pathological evaluation. Degrees of lymphatic vessel invasion were classified as 0 , no invasion; 1 , mild invasion; 2 , moderate invasion and 3 , severe invasion. The modes of invasive growth pattern were classified into two groups, namely expanding type, the overall pushing growth type of adenocarcinoma with a clear invasive margin; and infiltrating type, a widespread streaming form of adenocarcinoma with an unclear borderline of the invasive front (Fig. 1). To evaluate the myofibroblast distribution of each case, we selected the paraffin-embedded specimen that showed three invasive lesions in each histological layer (SM, MP and SS) as diagnosed by H\&E staining (Fig. 2).

Immunohistochemistry. For immunohistochemical examination regarding the myofibroblast distribution in each case, the paraffin-embedded specimen which was described in 'Pathological analysis' was a representative specimen of each case, and we used serial 4- $\mu \mathrm{m}$ sections for the immunohistochemical analysis. The sections were mounted on saline-coated glass slides. The antibodies used included $\alpha$-smooth muscle actin ( $\alpha$-SMA, 1:100, clone 1A4) and desmin (1:100, clone D-33) (both from Dako, Glostrup, Denmark). Immunostaining for $\alpha$-SMA and desmin was performed using the standard avidin-biotin-peroxidase complex method with an automated immunostainer (Benchmark XT; Ventana Medical System, Tucson, AZ, USA). The signature characteristic of myofibroblasts is an $\alpha$-SMA-positive and desmin-negative pattern, whereas that of smooth muscle is an $\alpha$-SMA-positive and desmin-positive pattern.

Image analysis. We used imaging analysis to investigate the myofibroblast density. All cases had an invasive lesion of the three colorectal walls: SM, MP and SS. To obtain the images, we used an Olympus microscope BX50 with a U PlanApo objective lens ( $x 4$ magnification), DP Control software and a DP-70 digital camera (all from Olympus, Tokyo, Japan). We applied ImageJ software (National Institutes of Health, Bethesda, MD, USA) to view and analyze our obtained images (19). We captured images of $\alpha$-SMA and desmin (Fig. 3A and D), and these images were binarised (Fig. 3B and E). The binarised images showed that the positively and negatively immunostained lesions were black and white, respectively. We made a subtraction image by pasting the binarised images of desmin 


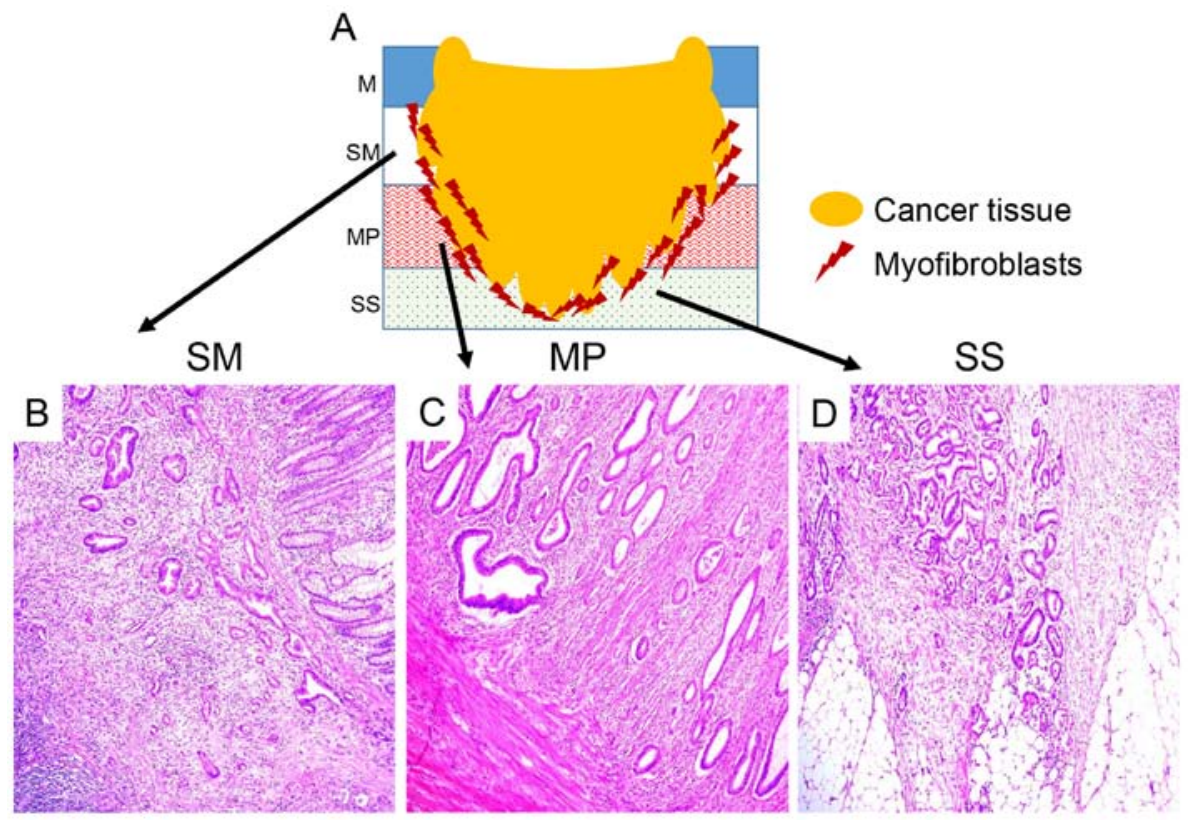

Figure 2. Representative case of the myofibroblast distribution in each invasive level of colorectal cancer. Myofibroblasts exist around the invasive front of each colorectal histological wall level (A). We identified the colorectal cancer invasive lesions using hematoxylin and eosin staining and selected the paraffinembedded specimen that showed three invasive lesions in each histological layer (SM, MP and SS) (B-D). M, mucosa; SM, submucosa; MP, muscularis propria; SS, subserosa.
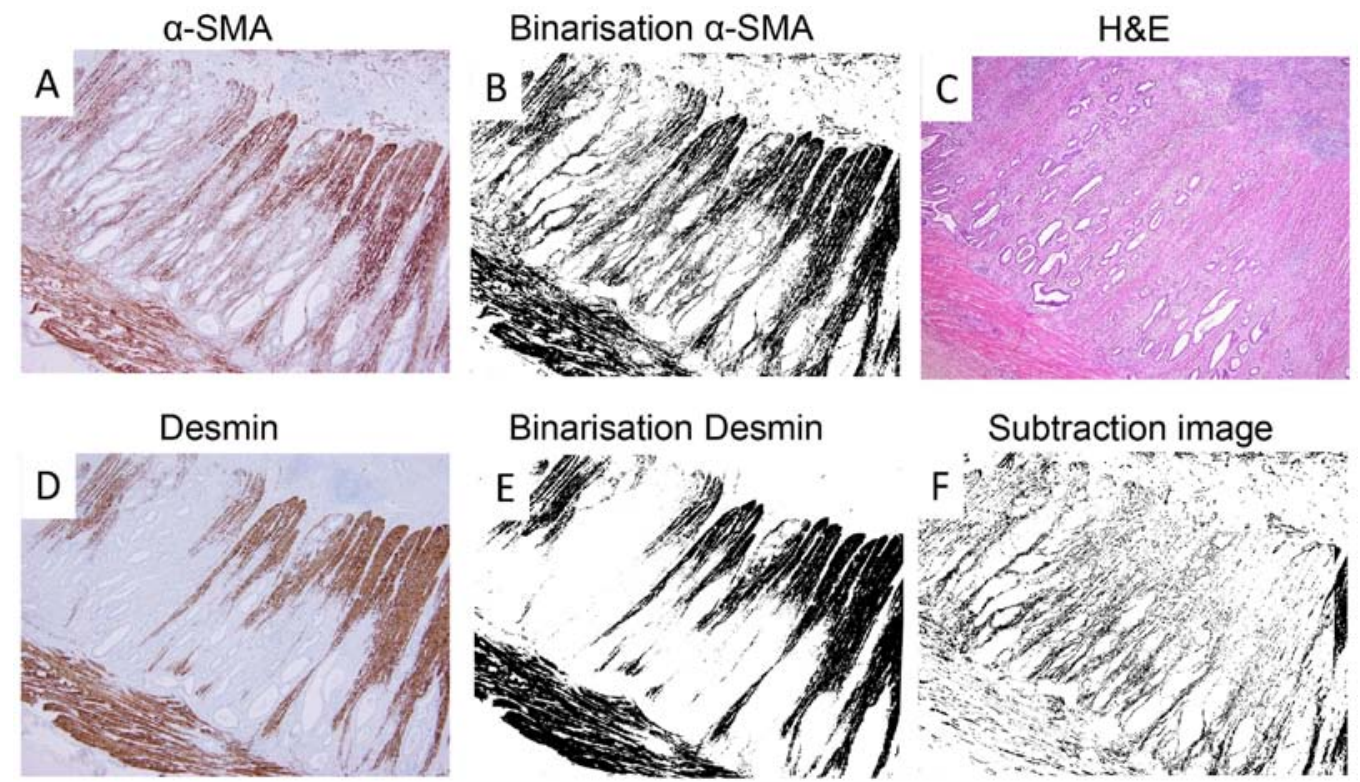

Figure 3. Representative case of an invasive lesion of the muscularis propria layer: $\alpha$-SMA (A), the binarisation image of $\alpha$-SMA (B), hematoxylin and eosin staining (C), desmin (D), the binarisation image of desmin (E) and subtraction image ( $\alpha$-SMA-desmin) (F). $\alpha$-SMA, $\alpha$-smooth muscle actin.

onto the binarised images of $\alpha$-SMA using the subtraction mode in ImageJ software (Fig. 3F). The subtraction images were shown as the value of $\alpha$-SMA minus that of desmin, and we could interpret the subtraction images showing myofibroblasts in the representative sections of each case. From all 121 cases, we obtained subtraction images of the three colorectal wall layers (SM, MP and SS) and measured the myofibroblast density in $1 \mathrm{x} 1 \mathrm{~mm}^{2}$ areas in the invasive border of each layer. We selected a hot spot myofibroblast density area from each invasive layer.
Statistical analysis. All values are presented as the means \pm standard error of the mean. Chi-square tests were performed for non-continuous variables, while the Mann-Whitney test and Welch t-tests were used for comparing continuous variables. Survival curves were constructed using the Kaplan-Meier method, and differences in survival were evaluated using the log-rank test. The relative prognostic factors were analysed with a Cox proportional hazards regression model. Differences were considered as statistically significant if the P-value was $<0.05$. Statistical analysis was performed with R (http://www.r-project. 
Table I. Histopathological characteristics of the 121 cases.

\begin{tabular}{|c|c|}
\hline Variables & No. of patients \\
\hline Age in years, median (range) & $67.4(26-93)$ \\
\hline \multicolumn{2}{|l|}{ Gender } \\
\hline Male & 66 \\
\hline Female & 55 \\
\hline \multicolumn{2}{|l|}{ Location } \\
\hline Colon & 77 \\
\hline Rectum & 44 \\
\hline \multicolumn{2}{|l|}{ Histological type } \\
\hline Well, mod & 110 \\
\hline Por, muc & 11 \\
\hline \multicolumn{2}{|l|}{ Invasive type } \\
\hline Expanding & 57 \\
\hline Infiltrating & 64 \\
\hline \multicolumn{2}{|l|}{ Lymphatic invasion } \\
\hline Low (ly0 or ly1) & 80 \\
\hline High (ly 2 or ly 3 ) & 41 \\
\hline \multicolumn{2}{|l|}{ Venous invasion } \\
\hline Low (v0 or v1) & 90 \\
\hline High (v2 or v3) & 31 \\
\hline \multicolumn{2}{|l|}{ Lymph node metastasis } \\
\hline Negative & 64 \\
\hline Positive & 57 \\
\hline
\end{tabular}

Well, well-differentiated adenocarcinoma; mod, moderately differentiated adenocarcinoma; por, poorly differentiated adenocarcinoma; muc, mucinous adenocarcinoma; ly, lymphatic invasion; v, venous invasion.

org) and Microsoft Excel software (Microsoft Corporation, Redmond, WA, USA).

\section{Results}

Clinicopathological characteristics. The clinicopathological characteristics of the 121 CRC cases are summarised in Table I. The series consisted of 66 men and 55 women, with a median age of 67.5 years (range, 26-93 years). The carcinomas were located in the colon (77 cases) and rectum (44 cases). One hundred and ten carcinomas were diagnosed as well and moderately differentiated adenocarcinoma, and 11 carcinomas were diagnosed as poorly differentiated and mucinous adenocarcinoma. In terms of the CRC invasive pattern, 57 cases were the expanding type, and 64 cases were the infiltrating type. Eighty cases and 41 cases had low and high degrees of lymphatic invasion, respectively. In contrast, the numbers of cases with low and high degrees of venous invasion were 90 and 31 cases, respectively. Furthermore, the numbers of cases with negative and positive lymph nodes were 64 and 57 cases, respectively.

Myofibroblast distribution in the invasive lesion at each colorectal wall stratified by expanding type vs. infiltrating type. We measured the myofibroblast density around the invasive front of each layer (SM, MP and SS) for the expanding and infiltrating types (Fig. 4A). In 57 cases of the expanding type, the mean myofibroblast densities for each wall of the invasive lesion were $11.03 \pm 0.88 \%$ (SM), $11.62 \pm 0.50 \%$ (MP) and $19.24 \pm 1.34 \%$ (SS). Meanwhile, in 64 cases of the infiltrating type, the mean myofibroblast densities were $13.60 \pm 0.79 \%$ (SM), $20.52 \pm 0.62 \%$ (MP) and $22.40 \pm 1.07 \%$ (SS). Significantly more myofibroblasts were located around these three invasive layers in the infiltrating type than the expanding type $(\mathrm{P}<0.05)$.

Association between the distributions of myofibroblast density and lymphatic vessel invasion. To investigate the association between the myofibroblast distribution and the degree of lymphatic vessel invasion, we stratified the 121 cases of CRC into either a low lymphatic vessel invasion (ly0 and ly1) group or a high lymphatic vessel invasion (ly2 and ly3) group. We analysed the myofibroblast distribution around the invasive front of each layer (Fig. 4B). The mean myofibroblast densities in the three layers within the low lymphatic vessel invasion group $(n=80)$ were $11.73 \pm 0.77 \%$ (SM), $13.89 \pm 0.57 \%$ (MP) and $19.99 \pm 1.06 \%$ (SS). In contrast, the mean myofibroblast densities in the high lymphatic vessel invasion group $(\mathrm{n}=41)$ were $14.68 \pm 1.39 \%(\mathrm{SM}), 21.48 \pm 1.05 \%(\mathrm{MP})$ and $24.76 \pm 1.86 \%$ (SS). The myofibroblast density was significantly higher in the group with high degree lymphatic vessel invasion than that noted in the group with low degree lymphatic vessel invasion in the MP $(\mathrm{P}<0.001)$ and SS layer $(\mathrm{P}=0.04)$, respectively. On the other hand, there was no significant difference between the low and high lymphatic vessel invasion group in regards to the myofibroblast density of the SM layer $(\mathrm{P}=0.103)$.

Association between the myofibroblast density distribution and venous vessel invasion. To investigate the association between the myofibroblast distribution and the degree of venous vessel invasion, we stratified the 121 cases into a low venous vessel invasion (v1 and v2) group and a high venous vessel invasion (v2 and v3) group and analysed the myofibroblast distribution around the invasive front of each layer (Fig. 4C). The mean myofibroblast densities in the low venous invasion group ( $\mathrm{n}=90)$ were $11.99 \pm 0.79 \%(\mathrm{SM}), 15.40 \pm 0.70 \%$ (MP) and $21.54 \pm 1.10 \%$ (SS), while the mean myofibroblast densities in the high venous invasion group $(n=31)$ were $14.85 \pm 1.47 \%$ (SM), $19.54 \pm 1.09 \%$ (MP) and $24.70 \pm 1.91 \%$ (SS). There was a significant difference in the myofibroblast density of the MP layer between the low and high venous invasion groups $(\mathrm{P}<0.01)$. There were not significant differences between the two groups in regards to the myofibroblast density of the SM layer $(\mathrm{P}=0.07)$ and $\mathrm{SS}$ layer $(\mathrm{P}=0.06)$.

Association between the myofibroblast distribution and lymph node metastasis. We stratified the $121 \mathrm{CRC}$ cases into a lymph node metastasis-negative group and -positive group and investigated the myofibroblast distribution of the three invasive walls (Fig. 4D). The mean myofibroblast densities of the three walls within the lymph node metastasis-negative group $(n=64)$ were $12.24 \pm 0.98 \%$ (SM), $14.12 \pm 0.63 \%$ (MP) and $20.73 \pm 1.16 \%$ (SS). The mean myofibroblast densities in the lymph node metastasis-positive group $(n=57)$ were $13.28 \pm 1.01 \%(\mathrm{SM})$, $19.01 \pm 0.97 \%(\mathrm{MP})$ and $22.61 \pm 1.56 \%$ (SS). The lymph 

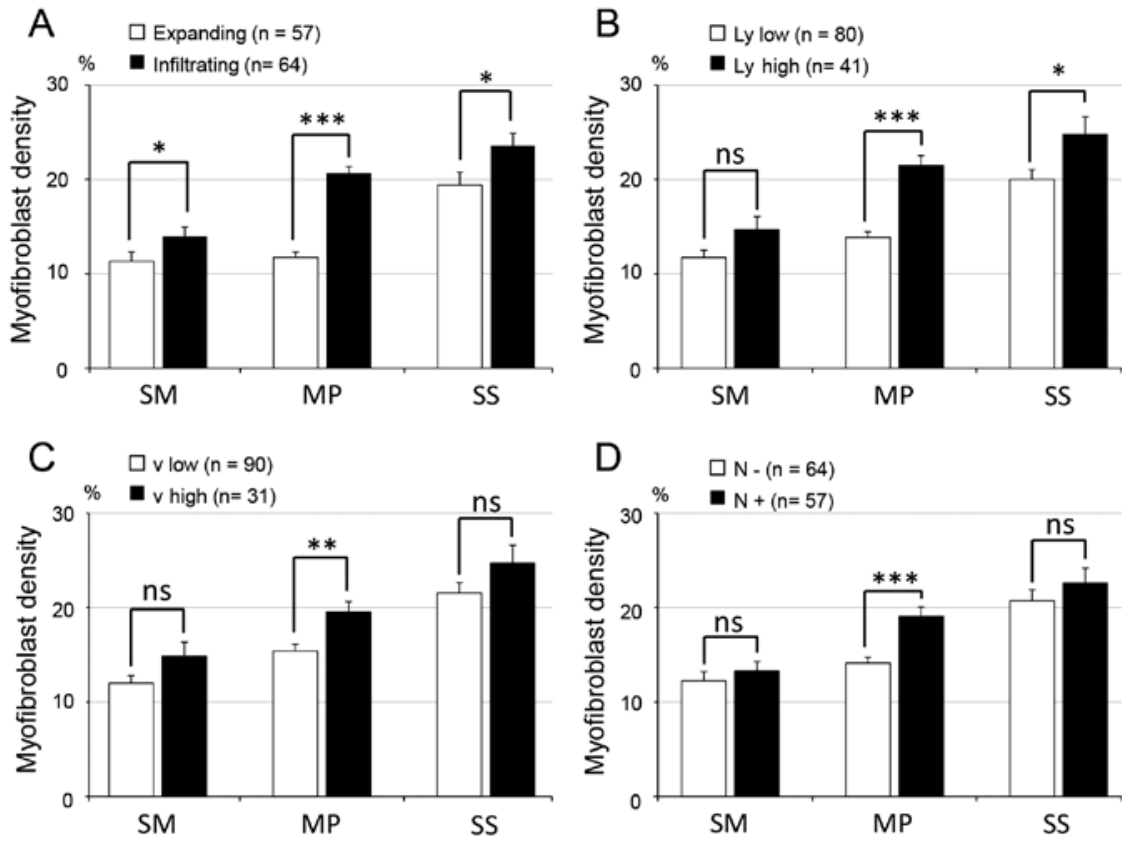

Figure 4. The mean myofibroblast density in each invasive level of colorectal cancer. The association between the invasive type (expanding and infiltrating) and myofibroblast distribution (A). The association between the lymphatic invasion and myofibroblast distribution (B). The association between venous invasion and myofibroblast distribution $(\mathrm{C})$. The association between lymph node metastasis and myofibroblast distribution (D). Values are given as the mean \pm standard error of the mean. ${ }^{*} \mathrm{P}<0.05,{ }^{* *} \mathrm{P}<0.01$ and ${ }^{* * * *} \mathrm{P}<0.001$. ns, not significant. $\mathrm{SM}$, submucosa; MP, muscularis propria; SS, subserosa.
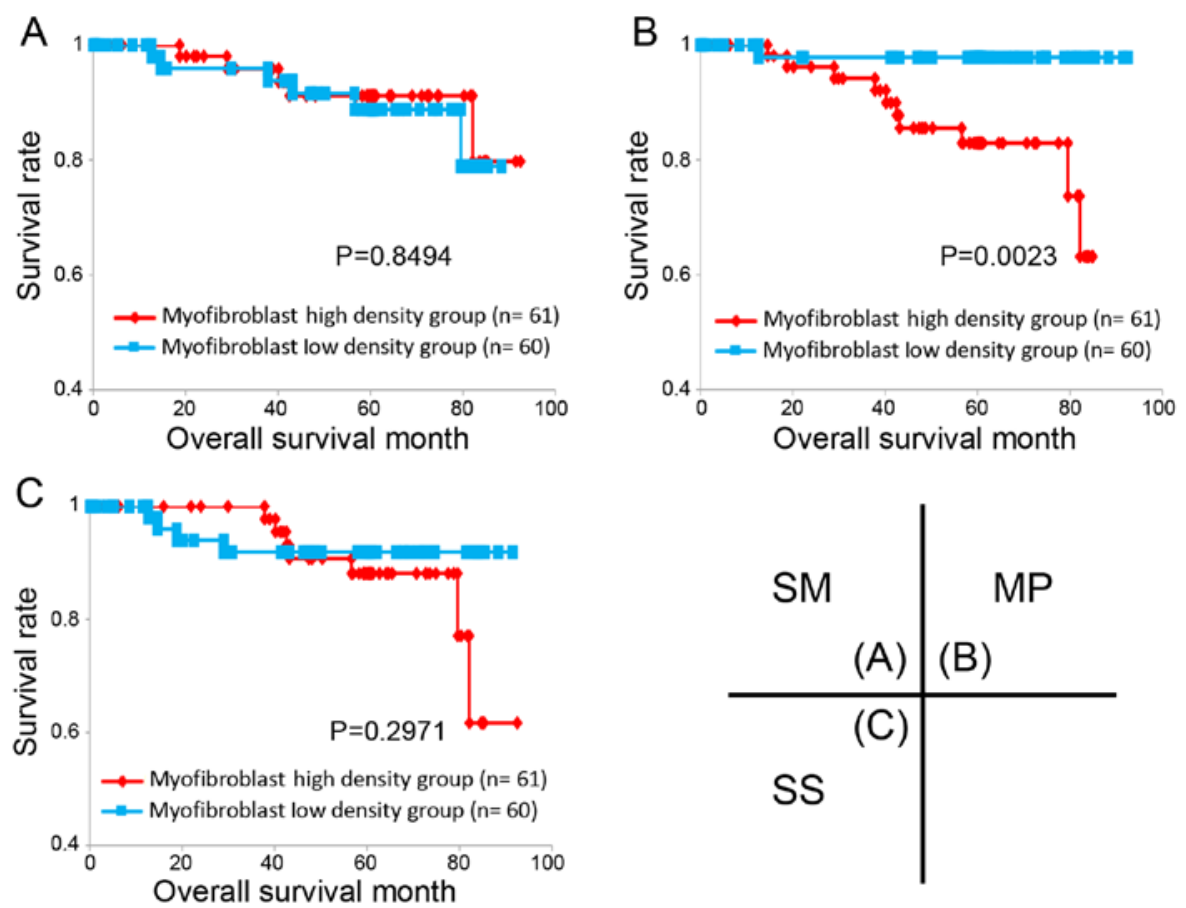

Figure 5. The association between the myofibroblast density of each invasive wall level and overall survival of patients with colorectal cancer. There was no significant difference between the high and low myofibroblast density groups in both the SM and SS invasive wall levels (A and C). There was a significant difference between the high and low myofibroblast density groups in the MP invasive wall level (B). SM, submucosa; MP, muscularis propria; SS, subserosa.

node-positive group had higher myofibroblast densities for all of the invasive layers than the lymph node-negative group. Furthermore, there was a significant difference between the lymph node metastasis-positive and -negative groups relating to the myofibroblast density of the MP layer $(\mathrm{P}<0.001)$. There were no significant differences between the two groups in regards to the myofibroblast density of the $\mathrm{SM}(\mathrm{P}=0.33)$ and SS layer $(\mathrm{P}=0.35)$.

Association between the myofibroblast density distribution and patient overall survival. To investigate the association between the myofibroblast distribution and overall survival, we 
Table II. Univariate and multivariate analyses of prognostic factors of survival.

\begin{tabular}{|c|c|c|c|}
\hline Variables & $\mathrm{n}(\%)$ & $\begin{array}{c}\text { Univariate } \\
\text { analysis } \\
\text { P-value }\end{array}$ & $\begin{array}{c}\text { Multivariate } \\
\text { analysis } \\
\text { P-value }\end{array}$ \\
\hline $\begin{array}{l}\text { SM myofibroblast } \\
\text { density }\end{array}$ & & 0.728 & - \\
\hline Low & $61(50.5)$ & & \\
\hline High & $60(49.5)$ & & \\
\hline $\begin{array}{l}\text { MP myofibroblast } \\
\text { density }\end{array}$ & & 0.025 & 0.332 \\
\hline Low & $61(50.5)$ & & \\
\hline High & $60(49.5)$ & & \\
\hline $\begin{array}{l}\text { SS myofibroblast } \\
\text { density }\end{array}$ & & 0.303 & - \\
\hline Low & $61(50.5)$ & & \\
\hline High & $60(49.5)$ & & \\
\hline Histological type & & 0.998 & - \\
\hline Well, mod & $110(90.9)$ & & \\
\hline Por, muc & $11(9.1)$ & & \\
\hline Invasive type & & 0.027 & 0.488 \\
\hline Expanding & $57(47.1)$ & & \\
\hline Infiltrating & $64(52.9)$ & & \\
\hline Lymphatic invasion & & 0.028 & 0.258 \\
\hline Low (ly0 or ly 1$)$ & $80(66.1)$ & & \\
\hline High (ly2 or ly 3 ) & $41(33.9)$ & & \\
\hline Venous invasion & & 0.392 & - \\
\hline Low (v0 or v1) & $90(74.4)$ & & \\
\hline High (v2 or v3) & $31(25.6)$ & & \\
\hline $\begin{array}{l}\text { Lymph node } \\
\text { metastasis }\end{array}$ & & 0.319 & - \\
\hline Negative & 64 (52.9) & & \\
\hline Positive & $57(47.1)$ & & \\
\hline
\end{tabular}

SM, submucosa; MP, muscularis propria; SS, subserosa; well, well-differentiated adenocarcinoma; mod, moderately differentiated adenocarcinoma; por, poorly differentiated adenocarcinoma; muc, mucinous adenocarcinoma. Mode of invasive type, as described in Materials and methods: ly, lymphatic invasion; $v$, venous invasion.

stratified the 121 cases of CRC into either a low myofibroblast density group or a high density group in each invasive layer and compared the high and low groups regarding the overall survival of the patients. The cut-off point between the two groups was set at the median value of the myofibroblast density in each invasive layer; the median values of the myofibroblast density were $11.52 \%$ in the SM layer, $16.19 \%$ in the MP layer and $21.48 \%$ in the SS layer. In only the MP level, but not the SM and SS layers, patients with high myofibroblast densities showed a significantly reduced overall survival ( $\mathrm{P}<0.003$; Fig. 5). To clarify the potential indicators, we analysed various pathological factors that were recorded in this study (Table II). Univariate analysis revealed that the following factors were correlated with poor prognosis: myofibroblast density of MP [relative risk (RR), 10.504; 95\% confidence interval (CI), 1.344-82.09; $\mathrm{P}=0.025$ ], invasive type (RR, 10.190; 95\% CI, 1.302-79.75; $\mathrm{P}=0.027$ ) and lymphatic invasion (RR, 4.4291; 95\% CI, 1.175-16.7; P=0.028). In the multivariate analysis, there was no significant difference among the myofibroblast density of the MP layer, the invasive type and lymphatic invasion.

\section{Discussion}

In the present study, we evaluated the association between clinicopathological characteristics of CRC and the myofibroblast distribution of three invasive layers using image analysis. We revealed that the myofibroblast density of MP plays an important role in CRC malignant behaviors, such as lymphatic invasion, venous invasion and lymph node metastasis, which can result in short overall survival of the patients.

We found that as the invasion of the CRC became deeper, the number of myofibroblasts increased around the invasive lesions, and the infiltrating growth type had a significantly higher density of myofibroblasts than that noted in the expanding type. Previous studies identified that the infiltrating type of CRC carries a high risk of liver metastasis and a worse prognosis compared with the expanding type (20-22). Myofibroblasts are a type of cancer-associated fibroblasts (CAFs) and are involved in desmoplastic reactions (23). CAFs actively associate with neoplastic cells and form the ECM of cancer lesions that promote cancer growth, angiogenesis and survival (24). CAFs interact with adjacent cancer cells through soluble factors or direct cell-cell adhesion to promote cancer cell invasion (25). In malignancy of CRC, myofibroblasts also promote CRC invasion and metastasis as they proliferate around the invasive lesion and alter the adhesive and migratory properties of CRC cells $(15,26)$. A previous study showed that myofibroblasts co-cultured with CRC cells may be involved in the invasiveness of CRC, even when the expression of E-cadherin, which is understood to be an adhesion molecule, prevents tumor cell invasiveness in vitro (27). Therefore, we suggest that it is possible that the large quantity of myofibroblasts which play a role as CAFs may alter both the adhesive and migratory properties of CRC cells and consequently aid CRC invasion into the deep colorectal layers. Moreover, our study indicated that the association between the infiltrating type, which is regarded as a malignant factor and myofibroblasts is stronger than the association between the expanding type and myofibroblasts.

Our results showed that the myofibroblast density of the MP layer was significantly higher in the group with a high frequency of lymphatic vessel and venous invasion compared with that in the group with a low frequency of lymphatic vessel and venous invasion. Furthermore, the lymph node-positive group had a significantly higher myofibroblast density in the MP layer than that of the lymph node-negative group. The lymphatic and venous vessels exist in three colorectal layers (SM, MP and SS), despite the differences in their histological structures. The distribution of lymphatic and venous vessels in normal colonic tissue tends to increase in frequency with depth throughout the wall (28). The functions of $\alpha$-SMA-positive myofibroblasts may be associated with promoting the ECM of tumor cells and lymphogenesis of the metastatic microenvironment in oral tongue squamous cell carcinoma (29). 
With respect to CRC, proliferation of myofibroblasts in the peri-tumoral areas was predicted to play an important role in lymphangiogenesis and was also found to be associated with lymph node metastasis (15). A previous study indicated that the CRC-invading MP layer may result in a greater ability to induce angiogenesis in adjacent normal tissue (30). Another study showed that the morphological mode of tumor invasion in the MP layer was associated with hematogenous metastasis of CRC (31). Our study predicted that compared to myofibroblasts of the other layers, myofibroblasts of the MP layer change the morphological mode of tumor invasion in CRC and increase the number of lymphatic and venous vessels that are invaded by CRC cells. Therefore, myofibroblasts of the MP layer are associated with the malignant potential of CRC, including lymph node metastasis.

The results of the univariate analysis revealed that myofibroblasts in the MP layer were significantly correlated with poor patient prognosis; however, the multivariate analysis using Cox proportional hazards model showed that a high myofibroblast density of MP was not an independent prognostic factor for overall survival. We suspected that the reason for this was that myofibroblasts of the MP layer may be strongly associated with the invasive growth pattern and lymphatic invasion.

In conclusion, we revealed that the myofibroblast distribution contributes to the malignant potential of CRC. Furthermore, we showed that myofibroblasts around the MP layer play an important role in the malignant potential and poor prognosis of CRC patients.

\section{Acknowledgements}

This study was supported by Grants-in Aid for Science from the Ministry of Education, Culture, Sports, Science, and Technology in Japan and a grant for Hirosaki University Institutional Research.

\section{References}

1. Ferlay JS, Ervik M, Diskshit R, Eser S, Mathers C, Rebelo M, Parkin D, Forman D and Baray F: GLOBOCAN 2012 v1.0, Cancer Incidence and Mortality Worldwide. IARC, 2012.

2. Sobin LH, Gospodarowicz MK and Wittekind C (eds). TNM Classification of Malignant Tumours. 7th edition. Wiley-Blackwell, 2009.

3. Seefeld PH and Bargen JA: The spread of carcinoma of the rectum: Invasion of lymphatics, veins and nerves. Ann Surg 118: 76-90, 1943.

4. Chapuis PH, Dent OF, Fisher R, Newland RC, Pheils MT, Smyth E and Colquhoun K: A multivariate analysis of clinical and pathological variables in prognosis after resection of large bowel cancer. Br J Surg 72: 698-702, 1985.

5. Shirouzu K, Isomoto H, Morodomi T, Araki Y and Kakegawa T: Lymphatic permeation of colorectal cancer - evaluation as a prognostic factor by prospective studies. Nihon Geka Gakkai Zasshi 92: 1686-1693, 1991 (In Japanese).

6. Royston D and Jackson DG: Mechanisms of lymphatic metastasis in human colorectal adenocarcinoma. J Pathol 217: 608-619, 2009.

7. van Netten JP, Cann SA and van der Westhuizen NG: Angiogenesis and tumor growth. N Engl J Med 334: 920-921, author reply 921, 1996.

8. Kim ER and Kim YH: Clinical application of genetics in management of colorectal cancer. Intest Res 12: 184-193, 2014

9. Ou J, Deng J, Wei X, Xie G, Zhou R, Yu L and Liang H: Fibronectin extra domain A (EDA) sustains CD133(+)/CD44(+) subpopulation of colorectal cancer cells. Stem Cell Res (Amst) 11: 820-833, 2013

10. Guan X: Cancer metastases: Challenges and opportunities. Acta Pharm Sin B 5: 402-418, 2015.
11. Seemayer TA, Schürch W and Lagacé R: Myofibroblasts in human pathology. Hum Pathol 12: 491-492, 1981.

12. Bissell MJ and Radisky D: Putting tumours in context. Nat Rev Cancer 1: 46-54, 2001.

13. Okamoto Y, Fujimori T, Ohkura Y, Sugai T, Arai T, Watanabe G, Wada R, Ueno H, Togashi K, Yao T, et al: Histological assessment of intra- and inter-institutional reliabilities in detection of desmoplastic reaction in biopsy specimens of early colorectal carcinomas. Pathol Int 63: 539-545, 2013.

14. Tsujino T, Seshimo I, Yamamoto H, Ngan CY, Ezumi K, Takemasa I, Ikeda M, Sekimoto M, Matsuura N and Monden M: Stromal myofibroblasts predict disease recurrence for colorectal cancer. Clin Cancer Res 13: 2082-2090, 2007.

15. Liang P, Hong JW, Ubukata H, Liu G, Katano M, Motohashi G, Kasuga T, Watanabe Y, Nakada I and Tabuchi T: Myofibroblasts correlate with lymphatic microvessel density and lymph node metastasis in early-stage invasive colorectal carcinoma. Anticancer Res 25: 2705-2712, 2005.

16. Yeung TM, Buskens C, Wang LM, Mortensen NJ and Bodmer WF: Myofibroblast activation in colorectal cancer lymph node metastases. Br J Cancer 108: 2106-2115, 2013.

17. Nakayama H, Enzan H, Miyazaki E, Naruse K, Kiyoku H and Hiroi M: The role of myofibroblasts at the tumor border of invasive colorectal adenocarcinomas. Jpn J Clin Oncol 28: 615-620, 1998.

18. Ueno H, Hase K, Hashiguchi Y, Ishiguro M, Kajiwara Y, Shimazaki $\mathrm{H}$ and Mochizuki $\mathrm{H}$ : Growth pattern in the muscular layer reflects the biological behaviour of colorectal cancer. Colorectal Dis 11: 951-959, 2009.

19. Schneider CA, Rasband WS and Eliceiri KW: NIH Image to ImageJ: 25 years of image analysis. Nat Methods 9: 671-675, 2012.

20. Morikawa T, Kuchiba A, Qian ZR, Mino-Kenudson M, Hornick JL, Yamauchi M, Imamura Y, Liao X, Nishihara R, Meyerhardt JA, et $a l$ : Prognostic significance and molecular associations of tumor growth pattern in colorectal cancer. Ann Surg Oncol 19: 1944$1953,2012$.

21. Rajaganeshan R, Prasad R, Guillou PJ, Chalmers CR, Scott N, Sarkar R, Poston G and Jayne DG: The influence of invasive growth pattern and microvessel density on prognosis in colorectal cancer and colorectal liver metastases. Br J Cancer 96: 1112-1117, 2007.

22. Jass JR, Love SB and Northover JM: A new prognostic classification of rectal cancer. Lancet 1: 1303-1306, 1987.

23. Karagiannis GS, Petraki C, Prassas I, Saraon P, Musrap N, Dimitromanolakis A and Diamandis EP: Proteomic signatures of the desmoplastic invasion front reveal collagen type XII as a marker of myofibroblastic differentiation during colorectal cancer metastasis. Oncotarget 3: 267-285, 2012.

24. Karagiannis GS, Poutahidis T, Erdman SE, Kirsch R, Riddell RH and Diamandis EP: Cancer-associated fibroblasts drive the progression of metastasis through both paracrine and mechanical pressure on cancer tissue. Mol Cancer Res 10: 1403-1418, 2012.

25. Yamaguchi H and Sakai R: Direct interaction between carcinoma cells and cancer associated fibroblasts for the regulation of cancer invasion. Cancers (Basel) 7: 2054-2062, 2015.

26. Martin M, Pujuguet $P$ and Martin F: Role of stromal myofibroblasts infiltrating colon cancer in tumor invasion. Pathol Res Pract 192: 712-717, 1996.

27. Dimanche-Boitrel MT, Vakaet L Jr, Pujuguet P, Chauffert B, Martin MS, Hammann A, Van Roy F, Mareel M and Martin F: In vivo and in vitro invasiveness of a rat colon-cancer cell line maintaining E-cadherin expression: An enhancing role of tumor-associated myofibroblasts. Int J Cancer 56: 512-521, 1994.

28. Duff SE, Jeziorska M, Kumar S, Haboubi N, Sherlock D, O'Dwyer ST and Jayson GC: Lymphatic vessel density, microvessel density and lymphangiogenic growth factor expression in colorectal cancer. Colorectal Dis 9: 793-800, 2007.

29. Ding L, Zhang Z, Shang D, Cheng J, Yuan H, Wu Y, Song X and Jiang $H$ : $\alpha$-Smooth muscle actin-positive myofibroblasts, in association with epithelial-mesenchymal transition and lymphogenesis, is a critical prognostic parameter in patients with oral tongue squamous cell carcinoma. J Oral Pathol Med 43: 335-343, 2014.

30. Fox SH, Whalen GF, Sanders MM, Burleson JA, Jennings K, Kurtzman S and Kreutzer D: Angiogenesis in normal tissue adjacent to colon cancer. J Surg Oncol 69: 230-234, 1998.

31. Kanno M, Kurosaka Y, Kosaka T, Yamaguchi A, Yonemura Y, Miwa K and Miyazaki I: Study on correlation of hematogenous metastasis in advanced colorectal cancer with the morphological mode of tumor invasion in the pm layer. Nihon Geka Gakkai Zasshi 93: 139-143, 1992 (In Japanese). 